\title{
Bounding the Set of Classical Correlations of a Many-Body System
}

\author{
Matteo Fadel ${ }^{1, *}$ and Jordi Tura ${ }^{2,3, \dagger}$ \\ ${ }^{1}$ Department of Physics, University of Basel, Klingelbergstrasse 82, 4056 Basel, Switzerland \\ ${ }^{2}$ ICFO-Institut de Ciencies Fotoniques, The Barcelona Institute of Science and Technology, 08860 Castelldefels (Barcelona), Spain \\ ${ }^{3}$ Max-Planck-Institut für Quantenoptik, Hans-Kopfermann-Straße 1, 85748 Garching, Germany
}

(Received 23 February 2017; published 6 December 2017)

\begin{abstract}
We present a method to certify the presence of Bell correlations in experimentally observed statistics, and to obtain new Bell inequalities. Our approach is based on relaxing the conditions defining the set of correlations obeying a local hidden variable model, yielding a convergent hierarchy of semidefinite programs (SDP's). Because the size of these SDP's is independent of the number of parties involved, this technique allows us to characterize correlations in many-body systems. As an example, we illustrate our method with the experimental data presented in Science 352, 441 (2016).
\end{abstract}

DOI: $10.1103 /$ PhysRevLett.119.230402

Introduction.-Local measurements on quantum systems can display correlations that may not be explained by any local hidden variable model (LHVM) [1] or, in other words, that cannot be reproduced by local deterministic strategies (LDS), even if assisted by shared randomness [2]. Bell inequalities bound the space of LHVM or "classical" correlations, and correlations that violate a Bell inequality are termed nonlocal. Besides their fundamental interest, nonlocal correlations are a key resource enabling novel quantum information processing tasks [3].

Finding all Bell inequalities is an extremely demanding problem [4], which is NP complete even in the bipartite scenario [5]. For this reason, complete lists of Bell inequalities exist only for the simplest scenarios, e.g., not beyond three parties [6-10].

In the multipartite case one needs to relax the condition of membership in the LHVM set, for example, by looking for Bell inequalities of a particular form. A natural choice is to exploit the symmetries of the physical system of interest [e.g., permutational invariance (PI) [11] or translational invariance (TI) [12]]. Moreover, to make them experimentally practical, it is desirable to ask for additional features such as being composed of low-order correlators only (see Refs. [13,14] and [12,15-17], for the PI and TI cases, respectively). In the PI case, this approach has recently allowed for the detection of Bell correlations in a BoseEinstein condensate of 480 atoms [18] and in a thermal ensemble of $5 \times 10^{5}$ atoms [19].

The usual technique to find Bell inequalities is via the characterization of the set of LHVM correlations. From a geometrical point of view this set is a polytope, i.e., a bounded convex set that can be described by a finite number of vertices, or, equivalently, by the intersection of a finite number of half-spaces. The vertices of the LHVM polytope can be listed, as they correspond to LDS, and from them the Bell inequalities defining the half-spaces can be computed. Unfortunately, going from one description to the other is extremely inefficient, due to the (double) exponential scaling of its complexity in the number of parties [20]. Restricting the search to Bell inequalities of a particular form results in (i) a better scaling $[12,14]$ and (ii) Bell inequalities that can be tested experimentally via collective observables, such as total spin components $[13,18,19]$. However, this approach of characterizing the LHVM polytope is still prohibitive when the number of parties is large, e.g., because a complete list of the vertices is inaccessible and because one has to infer classes of inequalities from a small number of parties [12-15], leaving potentially useful ones undiscovered.

In this work, we present a technique to approximate the set of LHVM correlations from the outside. Our technique can be seen as checking all Bell inequalities of a specific form with a single test. It is based on a hierarchy of semidefinite programs (SDP's) aproximating convex hulls of semialgebraic sets [21-24]. One key advantage of our method is that, contrary to other existing SDP's hierarchies $[25,26]$, the number of parties enters as a parameter in the SDP's, without affecting their size. Moreover, in all cases we considered, our hierarchy shows convergence to a relaxation of the LHVM set already at its lowest level. In summary, the method we propose provides an efficient sufficient condition for a set of correlations to be nonlocal, and it yields a certificate of nonlocality by providing the Bell inequality that is violated.

Overview of the method.-We consider the Bell scenario in which each of $N$ observers performs one of $d$ possible measurements on their share of the system. For simplicity, we are going to assume that every measurement yields outcomes \pm 1 , keeping the generalization to more outcomes for later. Let $\mathcal{M}_{k}^{(i)}$ be the $k$ th measurement of the $i$ th party. We are interested in Bell correlations that can be revealed by PI Bell inequalities composed of at most $K$-body correlators, i.e., inequalities of the form

$$
\sum_{k \leq K} \sum_{j_{1} \leq \ldots \leq j_{k}} \alpha_{j_{1} \ldots j_{k}} \mathcal{S}_{j_{1} \ldots j_{k}}+\beta_{C} \geq 0
$$


where $\alpha_{j_{1} \ldots j_{k}} \in \mathbb{R}, \beta_{C} \in \mathbb{R}$ is the so-called classical bound, and $\mathcal{S}_{j_{1} \ldots j_{k}}$ are the PI correlators defined as

$$
\mathcal{S}_{j_{1} \ldots j_{k}}=\sum_{\substack{i_{1} \ldots i_{k}=1 \\ \text { allis different }}}^{N}\left\langle\mathcal{M}_{j_{1}}^{\left(i_{1}\right)} \ldots \mathcal{M}_{j_{k}}^{\left(i_{k}\right)}\right\rangle .
$$

Our task is to certify that given experimental data $\overrightarrow{\mathcal{S}}_{K}^{*}=$ $\left(\mathcal{S}_{0}^{*}, \mathcal{S}_{1}^{*}, \ldots\right)$ cannot be described by LHVM, or, in other words, we want to construct a Bell inequality of the form of Eq. (1) that is violated for $\overrightarrow{\mathcal{S}}_{K}^{*}$, thus confirming the presence of Bell correlations. In order to achieve this, we perform two mild relaxations of the membership conditions to the LHVM set, which we show to become negligible as $N$ increases. This yields a hierarchy of SDP's of the form

$$
\begin{aligned}
\max _{y_{j} \in \mathbb{R}} 1 & \\
\text { s.t. } \tilde{\Gamma}(\vec{y}) & \succeq 0 \\
y_{0} & =1 \\
y_{j} & =\left(\overrightarrow{\mathcal{S}}_{K}^{*}\right)_{j},
\end{aligned}
$$

where $y_{0}$ and the $y_{j}$ corresponding to $\overrightarrow{\mathcal{S}}_{K}^{*}$ are fixed, while the remaining $y_{j}$ are free real parameters that can be varied until the moment matrix $\tilde{\Gamma}$ (that we are going to show how to construct) is positive semidefinite. If the SDP (3) is infeasible, its dual yields the coefficients $\alpha_{j_{1} \ldots j_{k}}$ and a (relaxed) classical bound $\beta_{C}$ of a Bell inequality of the form of Eq. (1) that certifies that $\overrightarrow{\mathcal{S}}_{K}^{*}$ is nonlocal. Note the crucial fact that the number of parties $N$ enters as a parameter in $\tilde{\Gamma}$, therefore, not affecting the size of the SDP. Our method can be seen as a way to check infinitely many Bell inequalities of the form of Eq. (1) at once. We now illustrate our method in more detail.

Details of the method.-If the observed statistics $\overrightarrow{\mathcal{S}}_{K}^{*}$ satisfy a LHVM, they belong to the LHVM polytope (projected) in the space of $K$-body symmetric correlators, $\mathbb{P}_{K}^{S}$. Its vertices correspond to LDS, satisfying

$$
\begin{aligned}
\left\langle\mathcal{M}_{j_{1}}^{\left(i_{1}\right)} \cdots \mathcal{M}_{j_{k}}^{\left(i_{k}\right)}\right\rangle & =\left\langle\mathcal{M}_{j_{1}}^{\left(i_{1}\right)}\right\rangle \cdots\left\langle\mathcal{M}_{j_{k}}^{\left(i_{k}\right)}\right\rangle \quad \text { (local), } \\
\left\langle\mathcal{M}_{j}^{(i)}\right\rangle & = \pm 1 \quad \forall i, j \quad \text { (deterministic) } .
\end{aligned}
$$

Fortunately, the PI condition reduces the number of vertices from exponential to polynomial in $N$, because only the amount of parties following the same LDS is relevant $[13,14]$. For this reason, it is natural to introduce $m=2^{d}$ variables $\vec{x}=\left(x_{1}, \ldots, x_{m}\right)$, where $x_{i}$ counts how many parties follow the $i$ th LDS. Note that the $x_{i}$ satisfy

$$
\sum_{i=1}^{m} x_{i}=N, \quad x_{i} \in \mathbb{Z}_{\geq 0} .
$$

Using this parametrization, at every vertex Eq. (2) can be written as a polynomial of degree $k$ in $m$ variables with real coefficients, i.e., $\mathcal{S}_{j_{1} \ldots j_{k}} \in \mathbb{R}[\vec{x}]_{k}$ (see Ref. [13] and the example). Denoting with $\overrightarrow{\mathcal{S}}_{K}$ the vector of such correlations up to degree $K$, we express $\mathbb{P}_{K}^{\mathrm{S}}$ as the convex hull $(\mathrm{CH})$ of $\overrightarrow{\mathcal{S}}_{K}$ evaluated on the parameter region defined by Eq. (6):

$$
\mathbb{P}_{K}^{\mathrm{S}}=\mathrm{CH}\left\{\overrightarrow{\mathcal{S}}_{K}(\vec{x}) \text { s.t. } \sum_{i} x_{i}=N, x_{i} \in \mathbb{Z}_{\geq 0}\right\} .
$$

First relaxation.-According to Eq. (7), $\mathbb{P}_{K}^{S}$ is defined as the convex hull of a finite set of points, therefore, not exploiting the inherent algebraic structure present in the polynomials $\overrightarrow{\mathcal{S}}_{K}(\vec{x})$. The first relaxation we introduce consists of dropping the condition $x_{i} \in \mathbb{Z}_{\geq 0}$, and considering instead $x_{i} \in \mathbb{R}_{\geq 0}$, which gives rise to the set

$$
\widetilde{\mathbb{P}_{K}^{S}}=\mathrm{CH}\left\{\overrightarrow{\mathcal{S}}_{K}(\vec{x}) \text { s.t. } \sum_{i} x_{i}=N, x_{i} \in \mathbb{R}_{\geq 0}\right\} \text {. }
$$

Note that $\overrightarrow{\mathcal{S}}_{K}(\vec{x})$ with $\vec{x} \in \mathbb{R}^{m}$ interpolates the vertices of $\mathbb{P}_{K}^{S}$, implying $\mathbb{P}_{K}^{S} \subseteq \widetilde{\mathbb{P}_{K}^{S}}$. As a consequence, if a set of correlations lies outside $\widetilde{\mathbb{P}_{K}^{S}}$, it also lies outside $\mathbb{P}_{K}^{S}$, and therefore it is nonlocal.

The correlators $\overrightarrow{\mathcal{S}}_{K}(\vec{x})$ can be expressed as a set of equations $f_{i}\left(\vec{S}_{K}\right)=0$, and similarly the non-negativity constraints $x_{j} \geq 0$ can be expressed as a set of constraints in $\overrightarrow{\mathcal{S}}_{K}$, by a set of inequalities $g_{j}\left(\overrightarrow{\mathcal{S}}_{K}\right) \geq 0$ (see the example). In what follows, we refer to the set of solutions of a system of polynomial equations $f_{i}\left(\overrightarrow{\mathcal{S}}_{K}\right)=0$ as an algebraic set. Moreover, if an algebraic set is further restricted by polynomial non-negativity constraints $g_{j}\left(\overrightarrow{\mathcal{S}}_{K}\right) \geq 0$, as is the case for $\widetilde{\mathbb{P}_{K}^{S}}$ in Eq. (8), we shall call such a set semialgebraic.

Second relaxation.-Deciding membership in the $\mathrm{CH}$ of a (semi)algebraic set $\mathcal{V}$ is NP hard [23]. However, there exist efficient approximations for $\mathrm{CH}(\mathcal{V})$ from the outside [21-24]. The idea behind these methods is to reduce the membership problem in $\mathrm{CH}(\mathcal{V})$ to that of a multivariate polynomial being non-negative, which can be relaxed to determining whether such polynomial can be expressed as a sum of squares (s.o.s.) [27]. While the first condition is NP hard, the second can be efficiently checked using a SDP, as we are going to show.

Following this approach, the main idea behind our method is to construct linear polynomials $l\left(\overrightarrow{\mathcal{S}}_{K}\right) \in \mathbb{R}\left[\overrightarrow{\mathcal{S}}_{K}\right]_{1}$ satisfying $l\left(\overrightarrow{\mathcal{S}}_{K}\right) \geq 0$ for all $\overrightarrow{\mathcal{S}}_{K} \in \mathcal{V}$, i.e., valid Bell inequalities defining half-spaces containing $\mathrm{CH}(\mathcal{V})$.

Starting from the observation that every polynomial of the form $p+\sum_{i} f_{i} p_{i}$, with $p, p_{i} \in \mathbb{R}\left[\overrightarrow{\mathcal{S}}_{K}\right]$, takes the same 
values when evaluated in $\mathcal{V}$ [because $f_{i}\left(\overrightarrow{\mathcal{S}}_{K}\right)=0$ for all $\overrightarrow{\mathcal{S}}_{K} \in \mathcal{V}$ ], we define the ideal $I$ generated by $f_{i}$ as the set

$$
I=\left\{\sum_{i} f_{i} p_{i} \text { s.t. } p_{i} \in \mathbb{R}\left[\overrightarrow{\mathcal{S}_{K}}\right]\right\} \subseteq \mathbb{R}\left[\overrightarrow{\mathcal{S}_{K}}\right],
$$

such that every polynomial in $p+I=\{p+q, q \in I\}$ is equivalent when evaluated in $\mathcal{V}$. Moreover, the ideal $I$ defines the set of equivalence classes $\mathbb{R}\left[\overrightarrow{\mathcal{S}_{K}}\right] / I$, where $p, q \in \mathbb{R}\left[\overrightarrow{\mathcal{S}_{K}}\right]$ are in the same class if they are equivalent modulo $I$, i.e., $p \equiv q \bmod I$, meaning that $p-q \in I$.

To express $l\left(\overrightarrow{\mathcal{S}}_{K}\right)$ we consider the following ansatz:

$$
l\left(\overrightarrow{\mathcal{S}}_{K}\right)=\sum_{i=0}^{m} g_{i}\left(\overrightarrow{\mathcal{S}}_{K}\right) \sigma_{i}\left(\overrightarrow{\mathcal{S}}_{K}\right) \quad \bmod I,
$$

where $g_{0}\left(\overrightarrow{\mathcal{S}}_{K}\right)=1$, and $\sigma_{i}\left(\overrightarrow{\mathcal{S}}_{K}\right)$ are s.o.s. polynomials modulo $I$ [i.e., there exists a s.o.s. polynomial in $\left.\sigma_{i}\left(\overrightarrow{\mathcal{S}}_{K}\right)+I\right]$. For compactness, let us use the shorthand notation $g_{i}$ and $\sigma_{i}$. Note that since all $g_{i} \geq 0$ in $\mathcal{V}$ by definition, and s.o.s. are non-negative, the form of Eq. (10) ensures the non-negativity of $l\left(\overrightarrow{\mathcal{S}}_{K}\right)$ in $\mathcal{V}$ [28].

Now, given a point $\overrightarrow{\mathcal{S}}_{K}^{*}$, our goal is to prove that $l\left(\overrightarrow{\mathcal{S}}_{K}^{*}\right)<0$ for some set of $\sigma_{i}$. If we succeed in this proof, then we have to conclude that $\overrightarrow{\mathcal{S}}_{K}^{*} \notin \mathrm{CH}(\mathcal{V}) \supseteq \mathbb{P}_{K}^{S}$, i.e., that the statistics in $\overrightarrow{\mathcal{S}}_{K}^{*}$ come from nonlocal correlations.

For computational reasons, we need to bound the maximum degree of the s.o.s. decomposition allowed in $\sigma_{i}+I$. The higher the degree, the larger the family of $l\left(\overrightarrow{\mathcal{S}}_{K}\right)$ that can be accessed through Eq. (10), but the more computationally expensive to produce such s.o.s. representation will be. This naturally yields a hierarchy of outer approximations to $\mathrm{CH}(\mathcal{V})$ by increasing the degree of the s.o.s. decomposition of $\sigma_{i}$. To simplify our exposition, we consider here the special case where all $\sigma_{i}=\sigma$.

To express all $\sigma$ that are s.o.s. of degree $2 \mu$, modulo $I$, we adopt the following procedure. First, we select (via a Gröbner basis [29]) a linearly independent set of representatives of $\mathbb{R}\left[\overrightarrow{\mathcal{S}}_{K}\right] / I$, and we order them in the vector $\vec{b}=\left(1, \mathcal{S}_{0}, \mathcal{S}_{1}, \ldots\right)^{T}$. Denoting by $\vec{b}_{\mu}$ the vector of elements of $\vec{b}$ of degree at most $\mu$, we write $\sigma=\sum_{j} s_{j}^{2} \bmod I$, where $s_{j}$ are linear combinations of the elements of $\vec{b}_{\mu}$; i.e., $s_{j}=\vec{b}_{\mu}^{T} \vec{a}_{j}$, with $\vec{a}_{j}$ real vectors. At this point, by defining the matrix $G=\sum_{j} \vec{a}_{j} \vec{a}_{j}^{T}$, which is positive semidefinite by construction $(G \succeq 0)$, and the moment matrix $\Gamma_{i}=g_{i} \vec{b}_{\mu} \vec{b}_{\mu}^{T}$ $\bmod I$, we write

$$
g_{i} \sigma=\Gamma_{i} \cdot G \quad \bmod I, \quad G \succeq 0 .
$$

Here, $X \cdot Y=\sum_{a b} X_{a b} Y_{a b}$.
When the elements of $\Gamma_{i}$ corresponding to $\overrightarrow{\mathcal{S}}_{K}$ are replaced by $\overrightarrow{\mathcal{S}}_{K}^{*}$, only some of its entries are constrained. If the remaining free parameters can be tuned to make $\Gamma_{i} \succeq 0$, Eq. (11) ensures that $g_{i} \sigma \geq 0$ in $\overrightarrow{\mathcal{S}}_{K}^{*}$ for all $\sigma$ (that are s.o.s. of degree $2 \mu$, modulo $I$ ). On the other hand, when $\Gamma_{i} \neq 0$ for any choice of the free parameters, there exists at least one $\sigma$ such that $g_{i} \sigma<0$ in $\overrightarrow{\mathcal{S}}_{K}^{*}$ [30].

Recall here that our final goal is to prove that there exists a $\sigma$ such that Eq. (10) gives $l\left(\overrightarrow{\mathcal{S}}_{K}^{*}\right)<0$. To this end, we write Eq. (10) as $l\left(\overrightarrow{\mathcal{S}}_{K}\right)=\tilde{\Gamma} \cdot \tilde{G} \bmod I$, where $\tilde{\Gamma}=\bigoplus_{i=0}^{m} \Gamma_{i}$, and similarly for $\tilde{G}$. As for Eq. (11), we ask whether $\tilde{\Gamma}$ can be made positive semidefinite at the point $\overrightarrow{\mathcal{S}}_{K}^{*}$. To perform this check with a SDP, we first reduce $\tilde{\Gamma}$ modulo $I$, and then linearize it as

$$
\tilde{\Gamma}=\sum_{j} y_{j} \tilde{\Gamma}_{j}
$$

where $y_{j}$ indexes the $j$ th element of $\vec{b}$, and $\tilde{\Gamma}_{j}$ are constant real matrices embodying the constraints among the entries of $\tilde{\Gamma}$. Now, for the point $\overrightarrow{\mathcal{S}}_{K}^{*}$, we execute SDP (3) to check the presence of nonlocal correlations.

If SDP (3) is infeasible, $\tilde{\Gamma} \neq 0$ independently on the free $y_{j}$, which proves that there exist a $\sigma$ such that $l\left(\overrightarrow{\mathcal{S}}_{K}^{*}\right)<0$, while $l\left(\overrightarrow{\mathcal{S}}_{K}\right) \geq 0$ for all $\overrightarrow{\mathcal{S}}_{K} \in \mathbb{P}_{K}^{S}$. Therefore, the infeasibility of SDP (3) certifies that $\overrightarrow{\mathcal{S}}_{K}^{*} \notin \mathrm{CH}(\mathcal{V}) \supseteq \mathbb{P}_{K}^{\text {S }}$, i.e., its nonlocal nature (see the example and Fig. 2).

While the output of SDP (3) is the answer feasible or infeasible, we can also write a SDP to maximize $\lambda$ subject to $y_{0}=1$ and $y_{j}=\lambda\left(\overrightarrow{\mathcal{S}}_{K}^{*}\right)_{j}$. The dual formulation of this modified SDP results in the dual variables $\alpha_{j_{1} \ldots j_{k}}$ associated to $y_{1} \ldots y_{i}$, and $\beta_{C}$ associated to $y_{0}$, defining a Bell inequality (1) that provides a certificate for the nonlocality of $\overrightarrow{\mathcal{S}}_{K}^{*}$ [31] (see the example and Fig. 2). In addition, maximizing $\lambda$ along different directions $\overrightarrow{\mathcal{S}}_{K}^{*}$ results in the points $\lambda_{\max } \overrightarrow{\mathcal{S}}_{K}^{*}$ that can be used to approximate the boundary of $\mathbb{P}_{K}^{S}$, (see Fig. 1).

On the other hand, if SDP (3) is feasible it means that it does not exist a $\sigma$ that is s.o.s. of degree $2 \mu$, modulo $I$, such that $l\left(\overrightarrow{\mathcal{S}}_{K}^{*}\right)<0$. In this case, we could access a higher level of our hierarchy by increasing $\mu$, which enlarges the class of $l\left(\overrightarrow{\mathcal{S}}_{K}\right)$ that can be represented [32].

An additional result in Refs. [21,22] ensures that, since the set $\mathcal{V}$ we want to approximate is compact, our hierarchy converges at least asymptotically to $\mathrm{CH}(\mathcal{V}) \equiv \widetilde{\mathbb{P}_{K}^{S}}$. Actually, in all examples we studied, we observed numerically that convergence at $\mu=1$ was already present.

Example.-In the spirit of Refs. [13,18], we consider $d=K=2$, giving rise to the set of correlators 


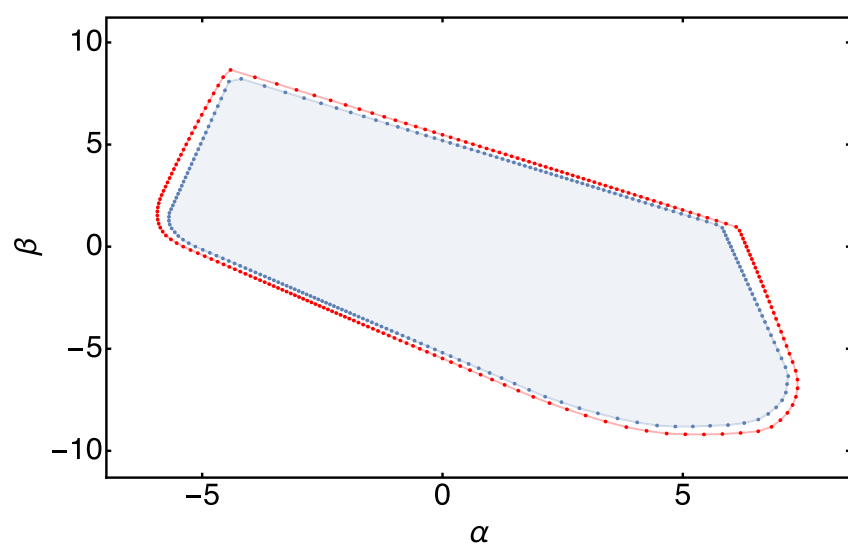

FIG. 1. For $N=10$ and $K=2$, the plane of the symmetric correlations of the form $\alpha \overrightarrow{\mathcal{S}}_{2}^{(1)}+\beta \overrightarrow{\mathcal{S}}_{2}^{(2)}$, with $\overrightarrow{\mathcal{S}}_{2}^{(1)}=$ $(1,-1,0,-1,1)^{T} / \sqrt{4}$ and $\overrightarrow{\mathcal{S}}_{2}^{(2)}=(0,-1,-1,1,0)^{T} / \sqrt{3}$. In blue, the intersection of $\mathbb{P}_{2}^{S}$ with the plane, computed with a linear program. In red, the boundary of the feasible set of SDP (3) for $\mu=1$. The gap between the two objects is imputable mainly to the first relaxation, and the small $N$ was chosen also to appreciate its size, which remains of the same order while $\mathbb{P}_{2}^{\mathrm{S}}$ increases with $N$ (see also Fig. 2).

$\overrightarrow{\mathcal{S}}_{2}=\left(\mathcal{S}_{0}, \mathcal{S}_{1}, \mathcal{S}_{00}, \mathcal{S}_{01}, \mathcal{S}_{11}\right) \in \mathbb{R}^{5}$, and $N$ parties. In this scenario, there are four LDS parametrized by $x_{i} \geq 0$ and satisfying $\sum_{i=1}^{4} x_{i}=N$. By expressing the correlators $\overrightarrow{\mathcal{S}}_{2}$ evaluated on a LDS in terms of $\vec{x}$, we obtain [13]

$$
\begin{aligned}
& \left(\begin{array}{l}
N \\
\mathcal{S}_{1} \\
\mathcal{S}_{0} \\
\mathcal{Z}
\end{array}\right)=\left(\begin{array}{c}
x_{1}+x_{2}+x_{3}+x_{4} \\
x_{1}+x_{2}-x_{3}-x_{4} \\
x_{1}-x_{2}+x_{3}-x_{4} \\
x_{1}-x_{2}-x_{3}+x_{4}
\end{array}\right), \\
& \left(\begin{array}{l}
\mathcal{S}_{00} \\
\mathcal{S}_{01} \\
\mathcal{S}_{11}
\end{array}\right)=\left(\begin{array}{c}
\mathcal{S}_{0}^{2}-N \\
\mathcal{S}_{0} \mathcal{S}_{1}-\mathcal{Z} \\
\mathcal{S}_{1}^{2}-N
\end{array}\right) .
\end{aligned}
$$

When $N$ is fixed Eqs. (13) are three free parameters, while Eqs. (14) define the ideal $I$, whose generators $\left\{f_{1}\left(\overrightarrow{\mathcal{S}}_{2}\right)\right.$, $\left.f_{2}\left(\overrightarrow{\mathcal{S}}_{2}\right)\right\}=\left\{\mathcal{S}_{00}-\mathcal{S}_{0}^{2}+N, \mathcal{S}_{11}-\mathcal{S}_{1}^{2}+N\right\}$ form also a Gröbner basis for $I$ [29]. Inverting Eq. (13) we obtain four polynomials in $\overrightarrow{\mathcal{S}}_{2}$ that allow us to express the constraints $x_{i}=g_{i}\left(\overrightarrow{\mathcal{S}}_{2}\right) \geq 0$, for instance,

$$
g_{1}\left(\overrightarrow{\mathcal{S}}_{2}\right)=\left[\mathcal{S}_{0}+\mathcal{S}_{1}+\left(\mathcal{S}_{0} \mathcal{S}_{1}-\mathcal{S}_{01}\right)+\left(\mathcal{S}_{0}^{2}-\mathcal{S}_{00}\right)\right] / 4 \geq 0 .
$$

At the first level of our hierarchy, $\mu=1$, the vector $\vec{b}_{1}^{T}=$ $\left(1, \mathcal{S}_{0}, \ldots, \mathcal{S}_{11}\right)$ generates the five $6 \times 6$ moment matrices $\Gamma_{i}$. Combined together, the $\Gamma_{i}$ give a $30 \times 30$ blockdiagonal moment matrix $\tilde{\Gamma}$, in which $N$ appears as a parameter, and thus not affecting its size.

Considering the experimental data presented in Ref. [18], we can conclude that the measured statistics

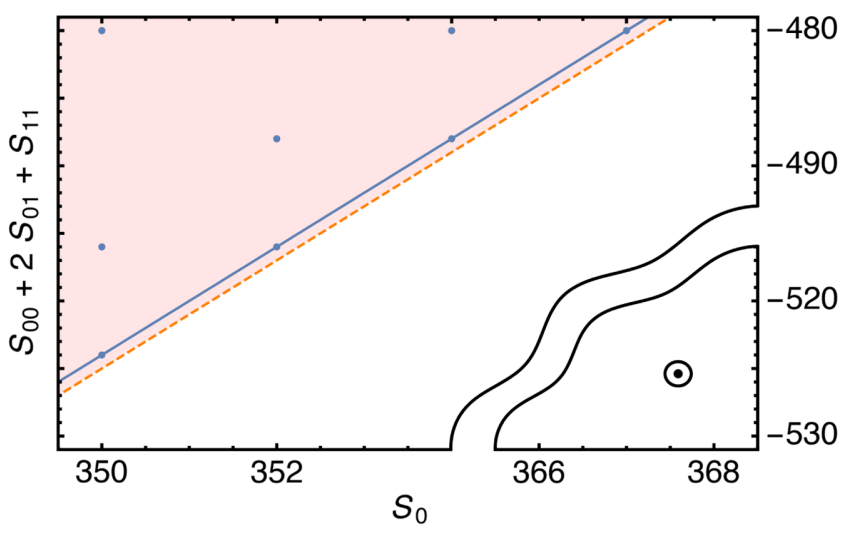

FIG. 2. Plane generated by $\left\{\mathcal{S}_{0},\left(\mathcal{S}_{00}+2 \mathcal{S}_{01}+\mathcal{S}_{11}\right)\right\}$. Black circled dot, point $(367.6,-525.4)$ measured experimentally in [18] for $N=476$. Blue points, projected vertices of $\mathbb{P}_{2}^{S}$. Blue line, bound given by the Bell inequality $-2 \mathcal{S}_{0}+\left(\mathcal{S}_{00}+2 \mathcal{S}_{01}+\mathcal{S}_{11}\right) /$ $2+2 N \geq 0$, from Refs. [13,18]. This inequality is tight, meaning that it is also a facet of the projected polytope. Pink region, points where SDP (15) gives $\lambda \geq 1$. Orange dashed line, Bell inequality obtained numerically by solving the dual of SDP (15). The distance between the blue and the orange lines is 1.000002 , meaning that the error of our method compared to the tight classical bound scales as $1 / N$, and it is imputable mainly to the first relaxation.

$\left(\mathcal{S}_{0}^{*}, \mathcal{S}_{00}^{*}+2 \mathcal{S}_{01}^{*}+\mathcal{S}_{11}^{*}\right)=(367.6,-525.4)$ contain Bell correlations because the following SDP gives $\lambda<1$ :

$$
\begin{aligned}
\max _{y_{j} \in \mathbb{R}} \lambda & \\
\text { s.t. } \tilde{\Gamma} & \succeq 0 \\
y_{0} & =1 \\
\left(y_{1}, y_{3}+2 y_{4}+y_{5}\right) & =\lambda\left(\mathcal{S}_{0}^{*}, \mathcal{S}_{00}^{*}+2 \mathcal{S}_{01}^{*}+\mathcal{S}_{11}^{*}\right) .
\end{aligned}
$$

The dual of SDP (15) gives as a result the dual variables associated to $y_{0}, y_{1}$, and $y_{3}+2 y_{4}+y_{5}$, which correspond, respectively, to the coefficients of the Bell inequality $\beta_{C}+\alpha_{1} \mathcal{S}_{0}+\alpha_{2}\left(\mathcal{S}_{00}+2 \mathcal{S}_{01}+\mathcal{S}_{11}\right) \geq 0$ (see Fig. 2).

Comment on more outcomes. - It is possible to consider the case where measurements have more outcomes by defining the expectation values as, e.g., $\left\langle\mathcal{M}_{j}^{(i)}\right\rangle^{(a)}=$ $2 P_{i}(a \mid j)-1$, where $P_{i}(a \mid j)$ is the probability that measurement $j$ on party $i$ gives as outcome $a$, and the symmetrized correlators as, e.g., $\mathcal{S}_{j}^{(a)}=\sum_{i=1}^{N}\left\langle\mathcal{M}_{j}^{(i)}\right\rangle^{(a)}$.

Conclusions. - We introduced a method to bound the set of LHVM correlations. Its main advantage, with respect to other techniques, is that there is no scaling with the number of parties, making it particularly suited for the study of nonlocal correlations in many-body systems. Our approach has several applications, some of which were presented here, such as the characterization of experimentally observed correlations or the derivation of new Bell inequalities. Furthermore, it can be easily generalized to scenarios with more measurements settings and outcomes, potentially 
enlarging the class of systems, and states, where nonlocal correlations could be experimentally detected.

We are grateful to Antonio Acín, Remigiusz Augusiak, Jean-Daniel Bancal, Gemma de las Cuevas, João Gouveia, and Philipp Treutlein for the useful discussions and comments on the manuscript. M.F. was supported by the Swiss National Science Foundation through Grant No. 200020_169591. J. T. was supported by Fundació Privada Cellex through the CELLEX-ICFO-MPQ programme, the Spanish MINECO (SEVERO OCHOA programme for Centres of Excellence in R\&D SEV-20150522 and National Plan FISICATEAMO FIS2016-79508-P), the Generalitat de Catalunya (SGR 874 and the CERCA programme), and EU grants OSYRIS (ERC-2013-AdG Grant No. 339106) and QUIC (H2020-FETPROACT2014 No. 641122). This project has received funding from the European Union's Horizon 2020 research and innovation programme under the Marie Skłodowska-Curie Grant Agreement No. 748549.

*matteo.fadel@unibas.ch jordi.tura@mpq.mpg.de

[1] J.S. Bell, On the Einstein-Podolsky-Rosen paradox, Physics 1, 195 (1964).

[2] A. Fine, Hidden Variables, Joint Probability, and the Bell Inequalities, Phys. Rev. Lett. 48, 291 (1982).

[3] N. Brunner, D. Cavalcanti, S. Pironio, V. Scarani, and S. Wehner, Bell nonlocality, Rev. Mod. Phys. 86, 419 (2014).

[4] L. Babai, L. Fortnow, and C. Lund, Non-deterministic exponential time has two-prover interactive protocols, Comput. Complex. 1, 3 (1991).

[5] D. Avis, H. Imai, T. Ito, and Y. Sasaki, Deriving tight Bell inequalities for 2 parties with many 2-valued observables from facets of cut polytopes, arXiv:quant-ph/0404014.

[6] D. Rosset, J.-D. Bancal, and N. Gisin, Classifying 50 years of Bell inequalities, J. Phys. A 47, 424022 (2014).

[7] J.F. Clauser, M. A. Horne, A. Shimony, and R. A. Holt, Proposed Experiment to Test Local Hidden-Variable Theories, Phys. Rev. Lett. 23, 880 (1969).

[8] S. Pironio, All Clauser-Horne-Shimony-Holt polytopes, J. Phys. A 47, 424020 (2014).

[9] I. Pitowsky and K. Svozil, Optimal tests of quantum nonlocality, Phys. Rev. A 64, 014102 (2001).

[10] C. Śliwa, Symmetries of the Bell correlation inequalities, Phys. Lett. A 317, 165 (2003).

[11] J.-D. Bancal, N. Gisin, and S. Pironio, Looking for symmetric Bell inequalities, J. Phys. A 43, 385303 (2010).

[12] J. Tura, A. B. Sainz, T. Vértesi, A. Acín, M. Lewenstein, and R. Augusiak, Translationally invariant multipartite Bell inequalities involving only two-body correlators, J. Phys. A 47, 424024 (2014).

[13] J. Tura, R. Augusiak, A. B. Sainz, T. Vértesi, M. Lewenstein, and A. Acín, Detecting nonlocality in many-body quantum states, Science 344, 1256 (2014).
[14] J. Tura, R. Augusiak, A. B. Sainz, B. Lücke, C. Klempt, M. Lewenstein, and A. Acín, Nonlocality in many-body quantum systems detected with two-body correlators, Ann. Phys. (Amsterdam) 362, 370 (2015).

[15] J. Tura, G. De las Cuevas, R. Augusiak, M. Lewenstein, A. Acín, and J. Ignacio Cirac, Energy as a Detector of Nonlocality of Many-Body Spin Systems, Phys. Rev. X 7, 021005 (2017).

[16] Z. Wang, S. Singh, and M. Navascués, Entanglement and Nonlocality in Infinite 1D Systems, Phys. Rev. Lett. 118, 230401 (2017).

[17] Z. Wang and M. Navascués, Two dimensional translationinvariant probability distributions: Approximations, characterizations and no-go theorems, arXiv:1703.05640.

[18] R. Schmied, J.-D. Bancal, B. Allard, M. Fadel, V. Scarani, P. Treutlein, and N. Sangouard, Bell correlations in a boseeinstein condensate, Science 352, 441 (2016).

[19] N. J. Engelsen, R. Krishnakumar, O. Hosten, and M. A. Kasevich, Bell Correlations in Spin-Squeezed States of 500000 Atoms, Phys. Rev. Lett. 118, 140401 (2017).

[20] B. Chazelle, An optimal convex hull algorithm in any fixed dimension, Discrete Comput. Geom. 10, 377 (1993).

[21] J. Gouveia, P. A. Parrilo, and R. R. Thomas, Theta bodies for polynomial ideals, SIAM J. Optim. 20, 2097 (2010).

[22] J. Gouveia and R. R. Thomas, Spectrahedral approximations of convex hulls of algebraic sets, in Semidefinite Optimization and Convex Algebraic Geometry (2012).

[23] J. B. Lasserre, Moments, Positive Polynomials and Their Applications, Series on Optimization and Its Applications Series (Imperial College Press, London, 2009).

[24] M. F. Anjos and J. B. Lasserre, Handbook on Semidefinite, Conic and Polynomial Optimization, International Series in Operations Research \& Management Science (New York, 2012).

[25] F. Baccari, D. Cavalcanti, P. Wittek, and A. Acín, Efficient Device-Independent Entanglement Detection for Multipartite Systems, Phys. Rev. X 7, 021042 (2017).

[26] M. Navascués, S. Pironio, and A. Acín, A convergent hierarchy of semidefinite programs characterizing the set of quantum correlations, New J. Phys. 10, 073013 (2008).

[27] Obviously, every s.o.s. polynomial is non-negative, but the converse is false in general. The textbook counter-example is the Motzkin polynomial, $x^{4} y^{2}+x^{2} y^{4}-3 x^{2} y^{2}+1$, which is non-negative on $\mathbb{R}^{2}$ but it is not a s.o.s. of elements of $\mathbb{R}[x, y]$.

[28] The modulo $I$ in Eq. (10) allows to reduce the degree of $l\left(\overrightarrow{\mathcal{S}}_{K}\right)$, potentially arriving to a linear polynomial.

[29] B. Buchberger, An algorithm for finding the basis elements of the residue class ring of a zero dimensional polynomial ideal, J. Symb. Comput. 41, 475 (2006).

[30] For a detailed proof, see the proof of Theorem 5.1 in Ref. [24].

[31] See Sec. 3.1 of Ref. [26] and, for a detailed derivation, Sec. 4.2 of Ref. [23].

[32] Note that the matrix $\tilde{\Gamma}$ for the level $\mu$, is a principal minor of the matrix $\tilde{\Gamma}^{\prime}$ for the level $\mu^{\prime}>\mu$. Therefore, if $\tilde{\Gamma}^{\prime} \succeq 0$ then necessarily $\tilde{\Gamma} \succeq 0$, while the converse is not always true. 